\title{
The Research Progress of Beef Cattle Intramuscular Fat Deposition Related Genes
}

\author{
Guijuan $\mathrm{Qu}^{1, \mathrm{a}}$, Shanghong Ding ${ }^{2, \mathrm{~b}}$ and Xiaoqing Dong ${ }^{1, c}$ \\ 1Jilin Agricultural University, College of animal science and technology, China \\ ${ }^{2}$ Jilin Teyan Biotechnological CoLLtd, Changchun 130122 \\ aquguijuan2003@sina.cn, b387867001@qq.com, ${ }^{\mathrm{c} d x g 200912 @ 163 . c o m}$
}

Keywords: Beef cattle; Intramuscular fat; Gene; Quality of beef cattle; Fat deposition

\begin{abstract}
Intramuscular fat content is an important factor to influence the quality of beef cattle, which directly affect the meat tender degree and flavor. For improve the quality of beef cattle through the increase intramuscular fat content, in this paper, the research progress of the beef cattle intramuscular fat deposition related gene are reviewed to provide reference for these functions gene further research .
\end{abstract}

\section{Introduction}

The content of intramuscular fat is a significant factor on the quality of beef cattle, which directly affects the meat tender degree and flavor. Currently, the content of intramuscular fat in beef cattle of our country is generally low, so how to improve the content of intramuscular fat to promote the quality of meat is crucial for the current study. A number of studies show that heredity is an important factor to affect the content of intramuscular fat, so genetic improvement is viable to improvement the content of IMF. Moreover, it is better for us to know IMF genetic mechanism, meanwhile accelerate to improve the quality of cow with the relevant gene of cow IMF deposition discovered recently. Therefore, this article overviews the advance of the relevant gene of cow IMF deposition to provide the basis of improving the meat quality.

\section{Fatty Acid-binding Proteins_-FABPs}

FABPs consist with 126-134 amino acid, the molecular weight is $14-16 \mathrm{kDa}$, in cytoplasm. The function of FABPs is about the transposition of fatty acid and some other hydrophobic ligands and the metabolism of lipid. It is the main research direction to increase the content of IMF for that they depositing triglyceride in myocardium and adipocyte, FABPs have many kinds of structures, there are two kinds of fatty acid-binding proteins were treated as IMF candidate gene: heart fatty acid binding protein and adipocyte-type fatty acid binding protein.

Heart Fatty Acid Binding Protein, H-FABP or FABPs. H-FABP gene is a $15 \mathrm{kD}$ protein, one of the most common types in FABP family. It expresses in myocardium, skeletal muscle, breast, and participates in transporting intracellular fatty acid which transports fatty acid from cell membrane to the position of fatty acid oxidation and triglyceride, phospholipid synthesis. H-FABP and fatty acid oxidation combine in the cells, which keep concentration difference intracellular and extracellular environment to make cell absorb fatty acid oxidation.

Quantity of studies showed, the expression quantity of FABP 3 affected by the factors of heredity and nutrition, and FABPs has a conspicuous influence on the tenderness of muscle and marbling. As Zhu's [1] reported on different kinds of beef cattle, with the dietary nutrients increased, the expression level increased, and affected the content of IMF to a certain degree, moreover, the FABP3 of Charlotte's hybrid cattle back fat has remarkable relativity on the expression of mRNA and IMF. Li[2] Xiang Xi cattle's gene polymorphisms existed remarkable relativity with the cattle intramuscular fat content and marbling, the type AA of marbling is significantly greater than the type of $\mathrm{AB}$ and $\mathrm{BB}$, and the type of $\mathrm{AB}$ and $\mathrm{BB}$ have little difference; Gene H-FABP can be one of intramuscular fat deposition candidate genes. Cao's[3] study showed that gene H-FABP impresses 
Yak eye muscle area and has consistent influence on intramuscular fat deposition, which suggested that gene H-FABP may be one of candidate genes affected by eye muscle area and Yak body weight. These studies showed that gene H-FABP has significantly influence on the marbling and the tender of beef cattle, so it can be one of intramuscular fat deposition candidate genes.

Adipocyte Fatty Acid Binding Protein, A-FABP. A-FABP is the family of FABPs. The A-FABP of mammal is not only the repository of triglyceride, which storage and release quantities of fatty acid on the forming and dissolving procedure of triglyceride, but also transport intracellular fat acid. Gene A-FABP only expressed in adipocyte, the gene A-FABP was located in number 14 of chromosome in cattle, has the length of $4391 \mathrm{bp}, 4$ exons, and the length of mRNA and CDS sequence respectively are $625 \mathrm{bp}$ and $339 \mathrm{bp}$.

Quantities of studies showed that A-FABP gene polymorphisms has markedly influence on intramuscular fat deposition [4]. Ji [5] analyzed Qin Chuan beef cattle gene A-FABP by bioinformatics, gene A-FABP of Qin Chuan beef cattle has 15 restriction Enzyme cutting sites, the molecule is $14.7 \mathrm{kDa}$, which consisted by132 amino acid, this correspond with that family FABPs usually has 126-137 amino acid and the molecule is14-16kDa. And it showed that Qin Chuan beef cattle A-FABP has the phosphorylation site and acylation site, and the character is likely to regulate lipid metabolism and ingest long chain fatty acid, and maintain internal environment homeostasis. Tang [6] studied Long Dong beef cattle, gene A-FABP has significantly influence on beef cattle shear force and dressing percentage.

\section{Adiponectin, AdipoQ}

Adiponectin, named Acrp 30, apM 1, GBP 28, AdipoQ, is a kind of steroids cytokine which is secreted by adipose tissue, it circulates in blood by the way of endocrine and regulates living body energy stability, glucose metabolism, and fat metabolism, it is the only fat cells specificity protein which negatively correlated to obesity. It is reported that adiponectin has many kinds of function, and the most significant function is to regulate fat metabolism, which plays an important role in body lipid metabolism regulation network.

As Ferguson [7] reported, adiponectin goes into blood circulation by impacting target tissue, it mainly activates AMPK to promote skeletal muscle fatty acid oxidation, and reduce lipid accumulation in skeletal muscle, decrease the dissociative FFA into liver to improve insulin resistance, lower the glycogenic and VLDL generation.

Adiponectin regulate and control fat metabolism by promote fat oxidation and restrain lipogenesis and promoting fat oxidation plays a leading role. Chen' [8] study showed that adiponectin can promote the expression of the key enzymes of ACO, L-FABP, CPTL, CPT II in the process of fat oxidation, increase fat $\beta$-oxidation and fat motivation. This can be the most mechanism for adiponectin regulates perinatal period dairy cattle. Zhang[9] discussed perinatal period dairy cattle different periods of adiponectin mRNA expression showed, the cattle adiponectin is closely related with fat metabolism and they found that adiponectin of fat cytokine can regulate and control fat metabolism by hormone and metabolic intermediates, and the gene expression of adiponectin is positively related to fat motivation key enzyme gene. Cong [10] research for the beef cattle, the level of ADPN mRNA is related with the content of intramuscular fat, which mentioned that adiponectin can be a beef cattle intramuscular fat candidate gene to participate fat metabolism.

\section{Melanocortinreceptor-4, MC4R}

Melanocortinreceptor-4 is coded by signal exon, a kind of peptides secreted by ventromedial hypothalamic nucleus. Quantities of MC4R lies in every corner in animal central neural system, which can regulate weight by central neural system, mainly present as feeding inhibition, reducing the body fat, decreasing the quality of body.

According to beef cattle, Liu's [11] study showed that genotype effect has great influence on the character of growth and fat deposition. Huang[12] studied MC4Rof Simon tal fattening cattle in 
China adipose tissue by the technology of Real time-PCR, the expression amount of MC4R in adipose tissue is obviously relative with back-fat thickness, with the back-fat thickness increased, the level of the expression of MC4R decreased, this is coincident with the physiological effect of restraining fat deposition. It is further proved that MC4R can be the important regulation factor to affect beef cattle fat deposition, which offered a theory basis for improving the quality of beef cattle.

\section{Fatty Acid Synthase, FAS}

In 1957, Wakil, et al. found FAS in goose liver homogenate. FAS catalyzed CoA and malony CoA combining fat acid in body to promote fat deposition. In the process of fat synthesis, with the activity of FAS increased, malony CoA compound of fatty acid with the catalyzed by FAS, which makes quantities of fatty acid deposition. Xiong's [13] study showed that the activity of FAS is obviously related to the amount of body fat, and the percentage of body fat in porcine adipose tissue. So the activity of FAS is important for controlling the deposition of fat. The expression level of FAS increased could significantly promote the deposition of triglyceride in body to lead fat. Qu's [14] study on the expression of FAS mRNA in beef cattle muscle showed that FAS is positively related with fat deposition, saying that FAS has great influence on the regulation of fat deposition. Yan's study about the influence of dietary nutrients on the expression of FAS showed that dietary fatty acid restrains the transcription of gene FAS, which makes protein decreased, and lower the combining of body fat. This confirmed that FAS is an important candidate gene for body fat deposition.

\section{Summary}

Fat trait is high heritability traits. Resent year, with the life style improved, people consciousness of health care enhance, we pay more attention on the content of fat in the body of human-beings and animals. So it is the crucial target for us how to improve the quality of meat. With the development of molecular biotechnology, the research for intramuscular fat deposition relevant gene offers theoretical foundation to regulate muscle fat.

\section{Reference}

[1] X.Y. Zhu, J.G. Wang and L.Y. Yang: The effect of nutrition level on FABP3 gene expression and IMF in crossbred beef cattle [J] Chinese Journal of Veterinary Science, Vol.31(2011)No.12, p.1781-1786. (in Chinese).

[2] Z.C. Li and K.L. Yi: Correlation analysis of Xiangxi Yellow Cattle H-FABP gene on marbling and intramuscular fat content [J] China Cattle Science, Vol.36(2010)No.1,p.1-4. (in Chinese).

[3] J. Cao:The correlation analysis of yak A-FABP and H-FABP gene and its association with carcass and meat quality traits [D] Lanzhou, Gansu Agricultural University,2012.

[4] J. Cao,Y.Z. Luo and J. Hu: The polymorphic analysis of yak fatty acid binding protein gen. Journal of Agriculture in North China, 2012, Vol.27(2012)No.4,p.42-47. (in Chinese).

[5] S.H. Ji, L.S. Qian and H.B Wang: The bioinformatics analysis of A-FAB P gene in Qin chuan Cattle. [J] Journal of Northwest agriculture and Forestry university, Vol.38(2010)No.6,p.77-82.

[6] H.J. Tang: Longdong cattle A-FABP and SCD1 gene polymorphism and its relationship with meat quality traits [D] Lanzhou, Gansu Agricultural University,2014.

[7] Ferguson J.F, Phillips C.M, Tierney A.C. et al: Gene-nutrient interactions in the metabolic syndrome: Single nucleotide polymorphisms in ADIPOQ and ADIPOR1 interact with plasma saturated fatty acids to modulate insulin resistance[J].The American Journal of Clinical Nutrition, Vol.91 (2010) No.3,p. 794-801. 
[8] H. Chen, L. Zhang and X.W. Li: The effect of adiponectin on the expression of key enzymes of lipid oxidation in the calf hepatocy [J].Chinese Journal of Veterinary Science,Vol.32(2012)No.10,p.1551-1555. (in Chinese)

[9] H. Zhang, P.Li and F. Yan: Different varieties of meat of bovine adiponectin expression and intramuscular fat deposition [J] Heilongjiang animal science and veterinary medicine, 2011,No.1,p.36-39.

[10] L.X.Cong, G.L.Zhang and P.Li: Bovine adiponectin and the relationship between intramuscular fat deposition [J] feed industry, Vol.35(2014)No.9,p.18-20.

[11] H. Liu, W. Tian and L.Z an,et al: Mutations of M C 4 Rgene and its association with economic traits in Qinchuan cattle [J] . MolBiolrep,2010,No.37,p.535-540.

[12] M. Huang, S.Z. Xu and J.Y. Li. Gene expression organization of Chinese Simmental MC3R and MC4R, and the correlation study in adipose tissue level and backfat thickness.[J] Journal of animal science and veterinary medicine,Vol.42 ( 2011) No.4,p.489-494. (in Chinese)

[13] W.Z.Xong and F. Yang: Study on growth hormone in different cross pigs fat metabolism [J ]Journal of animal science and veterinary medicine, 2001,Vol.32(2001)No.1,p.1-4 (in Chinese)

[14] G.J. Qu and X.Q. Dong: FAS mRNA expression of different hybridized combination beef cattle and its effect on intramuscular fat deposition[J]. Chinese journal of veterinary science, Vol.36 (2016) No.7,p.1183-1185,1211. (in Chinese). 